\title{
Water Contaminants Detection Using Sensor Placement Approach in Smart Water Networks
}

Essa Q. Shahra, Wenyan Wu

\begin{abstract}
Incidents of water pollution or contamination have occurred repeatedly in recent years, causing significant disasters and negative health impacts. Water quality sensors need to be installed in the water distribution system (WDS) to allow real-time water contamination detection to reduce the risk of water contamination. Deploying sensors in WDS is essential to monitor and detect any pollution incident at the appropriate time. However, it is impossible to place sensors on all nodes of the network due to the relatively large structure of WDS and the high cost of water quality sensors. For that, it is necessary to reduce the cost of deployment and guarantee the reliability of the sensing, such as detection time and coverage of the whole water network. In this paper, a dynamic approach of sensor placement that uses an Evolutionary Algorithm (EA) is proposed and implemented. The proposed method generates a multiple set of water contamination scenarios in several locations selected randomly in the WDS. Each contamination scenario spreads in the water networks for several hours, and then the proposed approach simulates the various effect of each contamination scenario on the water networks. On the other hand, the multiple objectives of the sensor placement optimization problem, which aim to find the optimal locations of the deployed sensors, have been formulated. The sensor placement optimization solver, which uses the EA, is operated to find the optimal sensor placements. The effectiveness of the proposed method has been evaluated using two different case studies on the example of water networks: Battle of the Water Sensor Network (BWSN) and another real case study from Madrid (Spain). The results have shown the capability of the proposed method to adapt the location of the sensors based on the numbers and the locations of contaminant sources. Moreover, the results also have demonstrated the ability of the proposed approach for maximising the coverage of deployed sensors and reducing the time to detect all the water contaminants using a few numbers of water quality sensors.
\end{abstract}

Keywords: Water distributed system, Water quality monitoring, Sensor placement, Optimization, Evolutionary algorithm;

Essa Q. Shahra

School of Engineering and Built Environment,

Birmingham City University, Birmingham, United Kingdom

Essa.Shahra@bcu.ac.uk

Tel: 44-121-3004291

Wenyan $\mathrm{Wu}$

School of Engineering and Built Environment,

Birmingham City University, Birmingham, United Kingdom

Wenyan.Wu@bcu.ac.uk

Tel: +44-121-3004291 


\section{Introduction}

The fundamental duties of water utilities are to supply and distribute water to customers in acceptable quality, quantity, and pressure through the entire water distribution system (WDS) (Liu et al. 2017). The usable water is at first treated to meet the standards of the drinking water quality before delivery. During transmission and dispersion, a few contaminants may enter WDS that adversely affect drinking water quality and cause health risks to customers (Du Plessis 2017; Kara et al. 2016).

The growth of infectious diseases begins with biological and chemical contaminants in drinking water (Cotruvo et al. 2018). Rapid and sensitive detection strategies are, therefore, crucial to guarantee a clean and safe water supply. Harmful supply of water affects human health, causing infectious illness, for example, influenza, hepatitis, gastric ulcers, pneumonia, and pulmonary disease (Richardson 2011). Water supplies contain various non-biological contaminants, including silica, sodium, sulphur, ammonia, and chlorine (De Vera and Wert 2019). In addition to these contaminants, the presence of microbiological contaminants in drinking water at the stage of consumption is another problem of water quality (Angelakis and Snyder 2015). Water utilities need to continually monitor both the quantity and quality of distributed water to maintain human health and improve safety (Shahra and Wu 2019).

WDS is typically comprised of numerous components to serve large zones with protected and constant supply of drinking water. They need regular administration, control, and maintenance (Gheisi et al. 2016). Therefore, monitoring water quality in WDS is not a simple job, and water utilities use traditional and advanced methods to complete this. In the conventional method, a group of water utility qualified employees visits the pre-determined monitoring sites for in situ water quality measurements and sampling at predefined frequencies (Capodaglio 2017). The data obtained using traditional monitoring provides discontinuous information on the monitored points of the water network. Thus, the detection and prevention of water contaminants are neither direct nor controlled. Alternatively, integrated inline sensor-based monitoring (ISBM) and supervisory control and data acquisition (SCADA) are an improved way to manage, maintain, control, and continuously monitor the quantitative and qualitative parameters of the system in many parts of a WDS (Zaev et al. 2016). As it is not possible to monitor all the parameters of water quality in the WDS, so it is necessary to balance the costs and the ease of use. As a result, ISBM systems typically use deployed sensors to measure hydraulic and typical water quality parameters such as electrical conductivity, turbidity, $\mathrm{pH}$, temperature, chlorine, and dissolved oxygen (Dong et al. 2015). Unlike traditional monitoring, the ISBM system with available sensors is always prepared and active to observe changes of the water quality in the WDS. With automatic sampling and measuring at prearranged time intervals, ISBM systems can cover as many points as required. The data gathered from the ISBM scheme is usually transmitted wirelessly to a control centre and stored for further use. Analytical techniques have recently taken a range of directions in water monitoring (Chen and Han 2018). There are multiple methods for monitoring water quality, including traditional instrumental analysis, microfluidic devices, model-based event detection, sensor placement approach, spectroscopic approach, and biosensors. The selection of an appropriate detection method depends heavily on the end of the planned detection analysis, whether quality or hybrid measurements are required. Chemical and biological sensors are in high demand that can be used in water monitoring technology and seems feasible for device inclusion and marketing (Karadirek et al. 2016; Thompson and Kadiyala 2014).

In recent years, the development of water quality sensors and the optimal placement of sensors for event detection has begun to develop (Zulkifli et al. 2018). A sensor placement approach can enhance detection efficiency by increasing sensitivity detection. The deployment of current water quality sensors in a WDS enables you to assess temperature, chlorine, $\mathrm{pH}$, turbidity, conductivity and dissolved oxygen (Chang et al. 2011). These parameters of water quality are essential for drinking water to fulfil the detection limit set by the United States Environmental Protection Agency (USEPA) (Rock et al. 2019). However, due to high demand, the installation of numerous sensors along the WDS does not seem to be very practical. Most of the water quality parameters are used as the leading indices for WDS contamination incidents, which are acquired from inline deployed sensors (De Winter et al. 2019). The primary aim of the water quality event detection technique is to: 1) define the water quality event in a subset (e.g., location, duration, and risk level), and 2) correctly and quickly detect water contamination (Zulkifli et al. 2018). The contribution of this paper can be summarised here: 
- Proposed a method for water quality sensor deployment that satisfied the requirement of the real-time monitoring for water quality in WDS by:

- Adapting the locations of the deployed sensors based on the number of suspected sources helping to reduce water contamination impact.

○ Maximized the coverage of the deployed sensors to detect all water contaminations in the study area.

- Reduce time detection of the water contaminations that helps to reduce the contamination impact.

The paper is organized as follows. Section 2 presents the related literature work, which has been done in the literature. Section 3 provides the methodology of the proposed work and more details of each part of the proposed approach. The evolutionary algorithm and the optimization objectives used to determine the optimal locations of the deployed sensors are explained as well. Section 4 presents the case studies applied to our approach for evaluating the effectiveness of the work. Section 5 shows the results, and the analysis of the sensor placements approach in both case studies are presented. Finally, section 6 concludes the work and recommend future works.

\section{Related Literature}

\subsection{Inline Sensor-Based Monitoring (ISBM)}

Wireless Sensor Networks (WSN), which can be utilized as a powerful solution to monitor and deal with water quality (Shahra et al. 2019). WSN has been generally executed for a full scope of applications, including observing, monitoring, and control (Sheltami et al. 2017). In WDS, many water quality sensors were installed to monitor contaminant incidents and to prevent colossal water contaminant accidents or to decrease water losses (Sela and Amin 2018). With the advent of increasing reliable computation in Microchips, many efficient sensors and other tools for real-time monitoring of remote regions are developed. Advances in wireless connectivity, distributed computing, and sensor technology now enable water quality data to be collected remotely, process, and communicate (Karem et al. 2017). Besides, integrating molecular and living monitoring technologies have enhanced ISBM systems with the capacity to react with a broader spectrum of contaminants and other parameters (Barabde and Danve 2015). ISBM technologies give many benefits over traditional monitoring methods by streamlining the data collection method, minimizing human errors and time delays, lowering general data collection costs, and considerably improving the amount and quality of temporal and spatial data (Adu-Manu et al. 2017). In general, inline water quality monitoring is described as gathering information at particular locations and at periodic intervals to provide data that can be used to define present water quality circumstances, and trends, etc. (Banna et al. 2014; Cloete et al. 2016). The primary objective of inline water quality monitoring in WDS is to assess critical water quality parameters such as chemical and physical characteristics, to detect variations of water parameters, and to recognize risks rapidly. Also, the monitoring system provides an analysis of the collected data in real-time and offers appropriate corrective actions (Zulkifli et al. 2018).

In recent years, incidents of water contaminants have often occurred and had adverse effects on our society. To ensure safe water is distributed, the water quality must be observed in real-time using a new technology that relies on the Internet of Things (IoT). Vinod et al. (2018) proposed water quality monitoring (WQM) based on IoT. WQM selects some of water quality parameters such as water level, $\mathrm{PH}, \mathrm{CO} 2$, and temperature across multiple system nodes. The data collected from these sensors are sent to the cloud server wirelessly. Within the cloud server, updated data can be retrieved or accessed for future analysis from anywhere in the world. Another study using the same technology of IoT to monitor the physical and chemical parameters of water quality provided the development and expansion of an Internet of Things real-time water quality measurement system and reduced the cost. It was used to measure the water's physical and chemical parameters such as conductivity, $\mathrm{pH}$, chlorine, and turbidity. The main benefit of the proposed work is that it is easy to install and can be installed very close to the target area (Yang et al. 2019).

Xiaoci Huang et al. (2015) presented a self-configuring WSN solution for the control of lake water quality. The methodology is based on the implementation of a semantic framework that allows a response to make decisions about configuring WSN services. They also proposed a rule-based reasoning system that is used by reasoning methods and context-awareness to support decision- 
making. They finally perform usability experiments and performance benchmarks to evaluate the approach. In Mompoloki Pule et al. (2016) WSN-based solution has been developed using Libelium's Waspmote platform. The sensor node is based on ATMEL's ATmega, which can run for a long time without the need for recharging its battery. The results showed that the proposed solution was able to sample specific water quality parameters in real-time with the same precision as expected. Another study that used WSN to design water quality parameters presented in Menon et al. (2012). They have developed and proposed a sensor framework to monitor water $\mathrm{pH}$ values and use such sensor nodes effectively to build a real-world water quality monitoring system in India. The measured value of $\mathrm{pH}$ is transmitted wirelessly to the base station using IEEE 802.15.4 communication standard for further processing and checking the condition of the water quality. The proposed framework provides multiple advantages such as low cost of sensor node platform, energyefficiency by using low power devices in the design of the hardware.

Tuna et al. (2013) have created two WSN methods to monitor water quality in drinking water tanks using sensors with distinct communication interfaces. In the first solution, they clarified the development of an autonomous mini-boat water quality monitoring framework for drinking water reservoirs, which is directed by GPS. Then they explored the use of portable IEEE 802.15.4-based wireless devices for continuous water quality monitoring. This kind of system removes the need for periodic water quality analysis and increases the quality of the water supply through constant monitoring. The second approach they have suggested uses a group of portable sensors with solar panels for energy harvesting and wireless devices installed on boats based on IEEE 802.15.4. These nodes communicate with each other wirelessly to form WSN and send their measurement to the central processing device for further analysis. The results of the first experiment show that the proposed solution has been successful in terms of direction and water sampling efficiency. The results of the second experiment show that the usefulness of this framework relies on multiple factors such as transmission capacity, packet size, and transmission rate.

\subsubsection{Deployment of Water Quality Sensors in WDS}

Deploying multiple sensitive sensor stations in a water distribution system is an emerging technology for the simultaneous detection of water contaminants. In recent years, multi-parameter sensor arrays are less expensive and easy to use for monitoring the quality of water supply systems (Zhuiykov 2012). To protect public people from the intentional and accidental penetration of risk into a distributed drinking water system, water quality monitoring sensors are deployed in WDS. The WDS can be presented as a network where the nodes represent water reservoirs, junction, tanks, pumps, and end-users, while the connections between these nodes represent the pipes. Contamination can occur at any node in the network and expand along the pipes to the entire system. Ideally, we can place sensors on all nodes to detect pollution or contamination. However, it is impossible to place sensors on all nodes of the network due to the relatively large structure of WDS and the high cost of water quality sensors (Rathi and Gupta 2014).

Speaking of the placement of water quality sensors, several design requirements must be taken into account, for example, to ensure maximum protection of public health, covering all possible water quality events, accessibility, etc. One of those issues is the cost of the deployment. This is because the prices of deployment and management are so high that it was impossible to place sensors covering the entire area, other than the critical places that have the most significant impact on protecting people. In response to growing concerns about water quality control, several studies have also analyzed location problems in water sensor networks, using mixed-integer programming models (MIP), heuristic methods, and general-purpose metaheuristics (Zeng et al. 2016).

Zeng et al. (2016) proposed a network of water quality monitoring systems comprising two types of systems at different rates. First, they formulate the problem into a programming problem that is limited by a mixed-integer quadratic. The problem is then linearized into a linear mixed-integer programming equivalent. They also propose a two-stage heuristic polynomial algorithm and test its efficiency through comprehensive studies based on simulations. Jiahong Chen et al. (2018) proposed Rapid Random Tree Exploration with Linear Reduction (RRLR). The method is based on an environmental model, and sensor readings are linearly dependent. It is also proved that the objective function of optimization, which uses the error of prior estimation, is submodular and leads to a nearoptimal solution. Simulation results show that, in comparison with benchmark algorithms, RRLR requires far fewer sensor nodes to achieve the same or lower estimation error. Ross et al. (2015) have presented a comparison and classification of a few water quality sampling methods for Canadian Prairies watersheds according to operational and statistical data. To detect and monitor video data on significant fields and water parameters including $\mathrm{pH}$, oxygen, temperature, dissolved, oxygen, and conductivity, a WSN-based water environment surveillance scheme is introduced in (Hall et al. 2007). In which data monitoring nodes send information and video to the database, and 
the base station transmits data to the remote monitoring centre using CDMA (Multiple Code Division Access) technologies. Lorena Parra et al. (2018), proposed a low-cost, wireless sensor network deployment system. The deployed sensors trace parameters of water quality, tank status, dropping feed, and swimming depth and speed of the fish. In addition, the sensor contains a smart algorithm to reduce the energy waste when sending the node information to the database. The monitor station consists of three nodes in each tank that send the data to a web-based database through the local area network and a smart algorithm that identifies irregular values and sends alarms when they occur. All sensors are designed, calibrated, and used to ensure their appropriateness. Storey et al. (2011) have discussed water quality parameters in the field of on-line monitoring of water quality, which presents the results of an international study on the detection of water pollution. The results are based on visits to major water utilities, research organizations and technology providers in Singapore, the United States, and Europe.

\subsubsection{Optimization Objective Functions for Sensor Deployment}

For the deployment of sensors in WDS, multiple objectives are currently being used. Minimizing effects on public health is a widely accepted objective, and many forms of goals have been developed on health impacts, such as the number of people exposed to a water contaminant or the number of people obtaining a dose above a fixed threshold. Researchers have also developed optimization methods for achieving a variety of other goals. For example, the time detection (TD), reducing the extent of contamination in the pipeline system (EC), population exposed to contamination (PE), volume of contaminated water consumed by the consumer before detection (VCW), and detection likelihood (DL), which is a method to calculate the value of one or more parameters for a given statistics, making the known probability distribution maximum. In Figure 1, we list the well-known and most used optimizations objectives (Rathi and Gupta 2014).

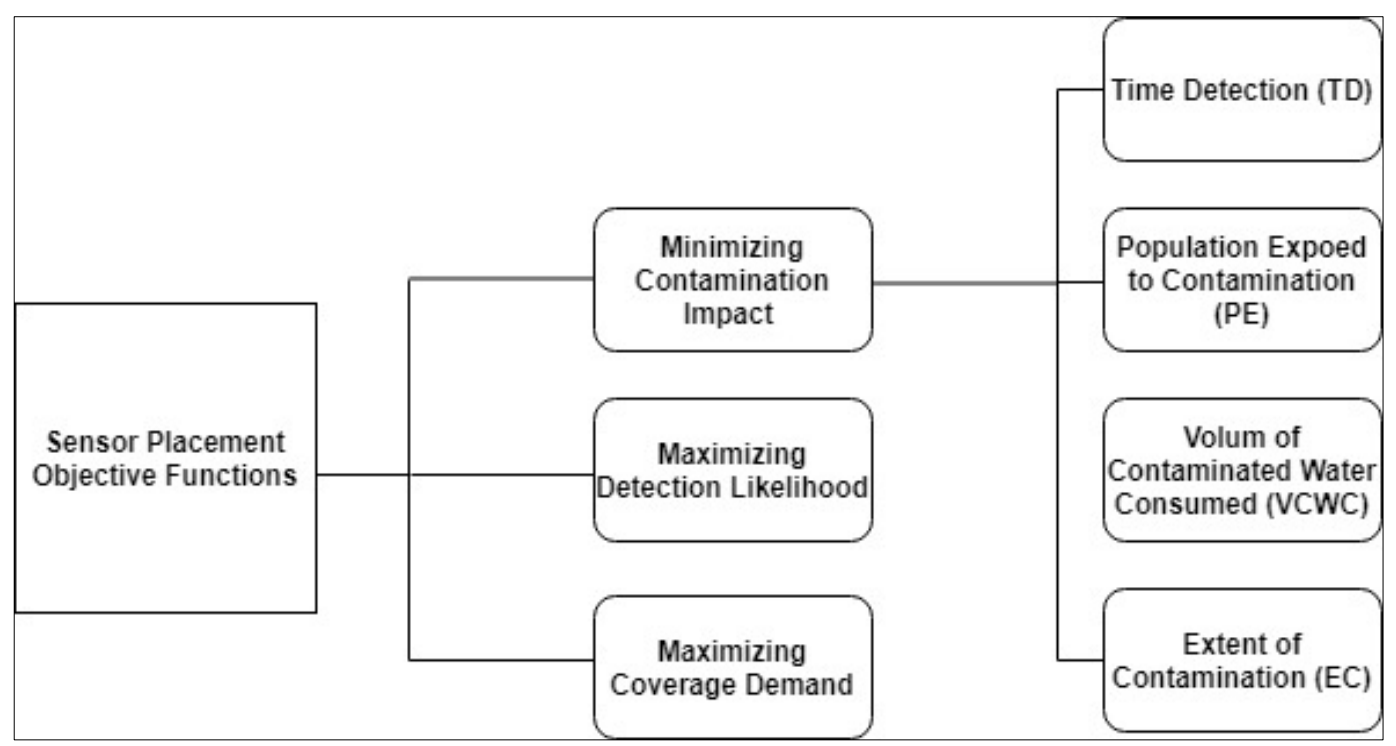

Figure 1 Objective functions used by seneor placement optimization

\subsection{Mathematical Formulation of WDS and Sensor Placement 2.2.1 System Model of WDS}

WDS, containing thousands of pipes, junctions, and hydraulic valves, may or may not have a loop or branch network topology, as shown in Figure 2. WDS is often modeled as a graph $\mathrm{G}=(\mathrm{N}, \mathrm{C})$, where the nodes in $\mathrm{N}$ refer to junctions, reservoirs, or other sources, and the connection in $\mathrm{C}$ represents pipes, valves, and pumps. Drinking water flow depends on pump rate and pressure, which may often change. A contaminant event refers to the injection of toxic substances as a result of water quality events that can occur at any node at any time with an undetermined dose of toxic chemicals or biological materials. The set of various contaminant scenarios is endless due to uncertainty; a representative set of situations (such as the contamination occurs at the water source, interconnection nodes, isolated nodes) can be selected either by listed or randomly for a quantities assessment of the damage. A water flow takes time to pass from one node to another, defined as the flow time. We use $t_{s d}$ to denote the average flow time from $s$ to $d$. The flow of $s$ can pass through several nodes until it reaches $d$. The average flow time can be obtained by adding the average time on all connections passed through. As contaminant flows along the water pipe, $t_{s d}$ also has time to spread the 
contaminant from node $s$ to node $d$. In particular, we assume $t_{s s}=0 \forall s \in \mathrm{N}$, which indicates that the contamination should immediately reach the junction at which it is introduced. To ensure detection accuracy for quick detection, any contamination injection must be detected by the sensor at time $T$. For example, if we place the sensor on node $S$, it can detect any possible contamination occur at node $S \in N$ with $t_{s d} \leq T$. We determine detectability $\left(d_{s d}\right)$ between nodes $s \in \mathrm{N}$ and $d \in \mathrm{N}$ as

$$
d_{s d}=\left\{\begin{array}{ll}
1, & \text { if } t_{s d} \leq T \\
0, & \text { otherwise }
\end{array} \quad s, d \in \mathrm{N} \quad\right. \text { (1) }
$$

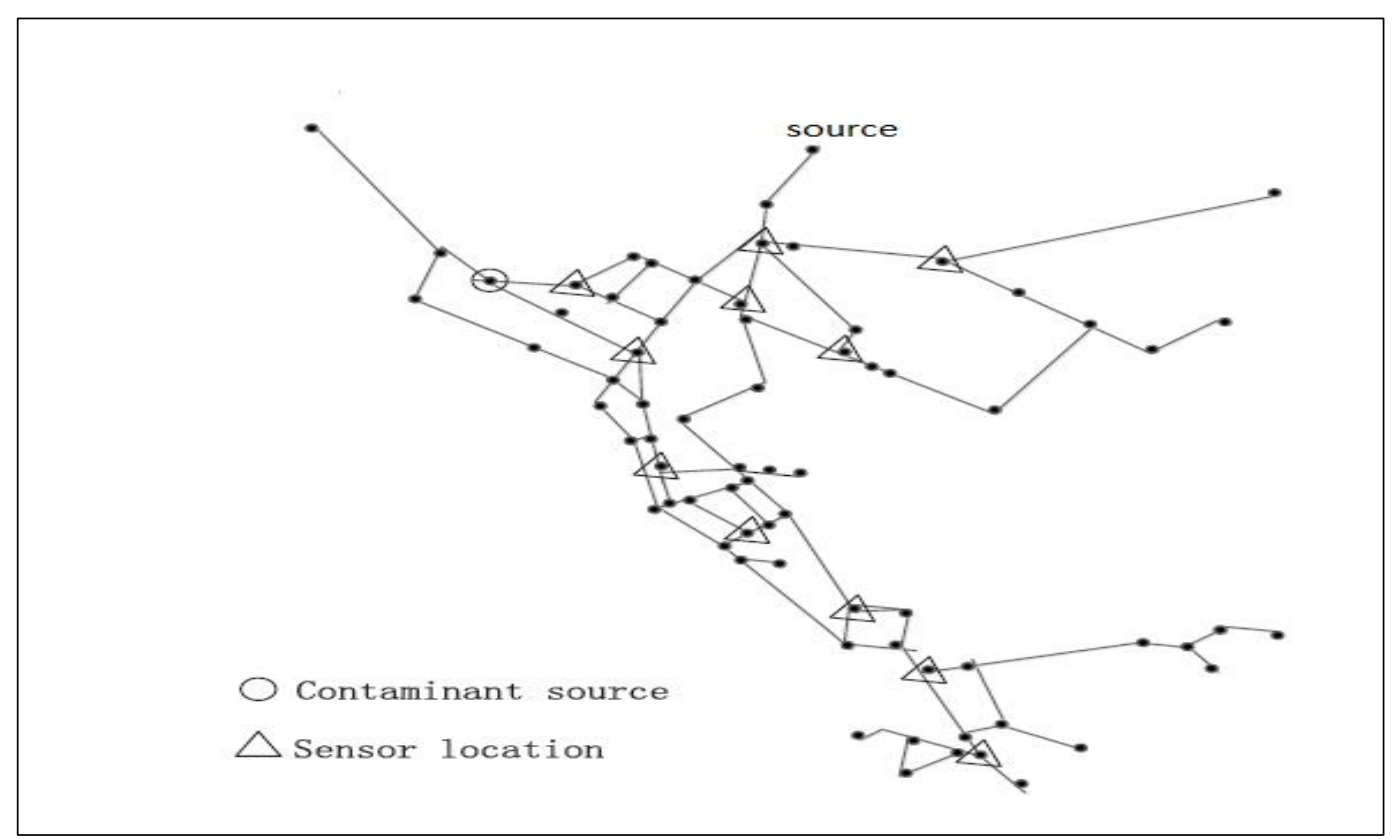

Figure 2 Water distribution system

\subsubsection{Sensor Placement Formulation}

Typically, the purpose of placing sensors in a WDS system is to detect contamination and thus mitigate the effects of contamination events. Here is a generally mixed integer programming (MIP) formulation for designing the expected impact sensor placement:

$$
\operatorname{Min} \sum_{\mathrm{e} \in \mathrm{A}} \alpha_{\mathrm{e}} \sum_{\mathrm{i} \in \mathrm{L}_{\mathrm{e}}} \mathrm{I}_{\mathrm{ei}} \mathrm{V}_{\mathrm{ei}}
$$

Constrains:

$$
\begin{array}{ll}
\text { 1. } \sum_{\mathrm{i} \in \mathrm{L}_{\mathrm{e}}} \mathrm{V}_{\mathrm{ei}}=1 & \forall_{\mathrm{e}} \in \mathrm{A} \\
\text { 2. } \mathrm{V}_{\mathrm{ei}} \leq \mathrm{S}_{\mathrm{i}} & \forall_{\mathrm{e}} \in \mathrm{A}, \mathrm{i} \in \mathrm{L}_{\mathrm{e}} \\
\text { 3. } \sum_{\mathrm{i} \in \mathrm{L}} \mathrm{C}_{\mathrm{i}} \mathrm{S}_{\mathrm{i}} \leq \mathrm{B} & \\
\text { 4. } \mathrm{S}_{\mathrm{i}} \in\{0,1\} & \forall_{\mathrm{i}} \in \mathrm{L} \\
\text { 5. } 0 \leq \mathrm{V}_{\mathrm{ei}} \leq 1 \quad \forall_{\mathrm{e}} \in \mathrm{A}, \mathrm{i} \in \mathrm{L}_{\mathrm{a}}
\end{array}
$$

This formulation is to minimize the expected effect of a set of contaminants events defined by (A). For each event $e \in A,\left(\alpha_{e}\right)$ is the weight of the event $(e)$, which is a probability of contamination injection. $\left(I_{e i}\right)$ is the health impact of the contamination event $(e)$ at node $(i) .\left(L_{e}\right)$ is the set of locations that can be contaminated by an event $(e) .\left(V_{e i}\right)$ is a decision variable that indicates whether the event $(e)$ detected (seen) by the sensor at the location $(i)$. It defined as a continuous variable between [0-1]. $\left(V_{i}\right)$ is a binary decision variable, $\left(S_{i}=1\right)$ indicates that a sensor deployed at node (i). $\left(C_{i}\right)$ is the cost of deploying a sensor at the location $(\mathrm{L})$. (B) is the budget available for using sensors. 


\section{Methodology}

\subsection{The Proposed Approach}

The proposed method presented in Figure 3. generates a multiple set of water contamination scenarios in several locations selected randomly in the WDS. Each contamination scenario spreads in the water networks for several hours, and then the proposed approach simulates the various effect of each contamination scenario on the water networks. On the other hand, the multiple objectives of the sensor placement optimization problem, which aim to find the optimal locations of the deployed sensors, have been formulated. The sensor placement optimization solver, which uses the EA, is operated to find the optimal sensor placements. In this research, the contaminated water consumed volume (VCW) and time detection (TD) are considered as optimization objectives. The novel aspect of the work described in this paper is that the number of contamination sources considered can vary. Thereby simulating scenarios where contamination occurs at one or two (or multiple - but not considered in this study) sources, and optimal sensor placements tailored to each of these scenarios are sought. In this context, the proposed approach described in this paper aims at providing the optimal sensor locations for efficiently detecting events when different numbers of contamination sources are suspected and maximizing the coverage of the deployed sensors to cover the whole network. More details of all the components of the proposed work: impact matrix and the optimizer solver are described in the next subsections 3.2 and 3.3.

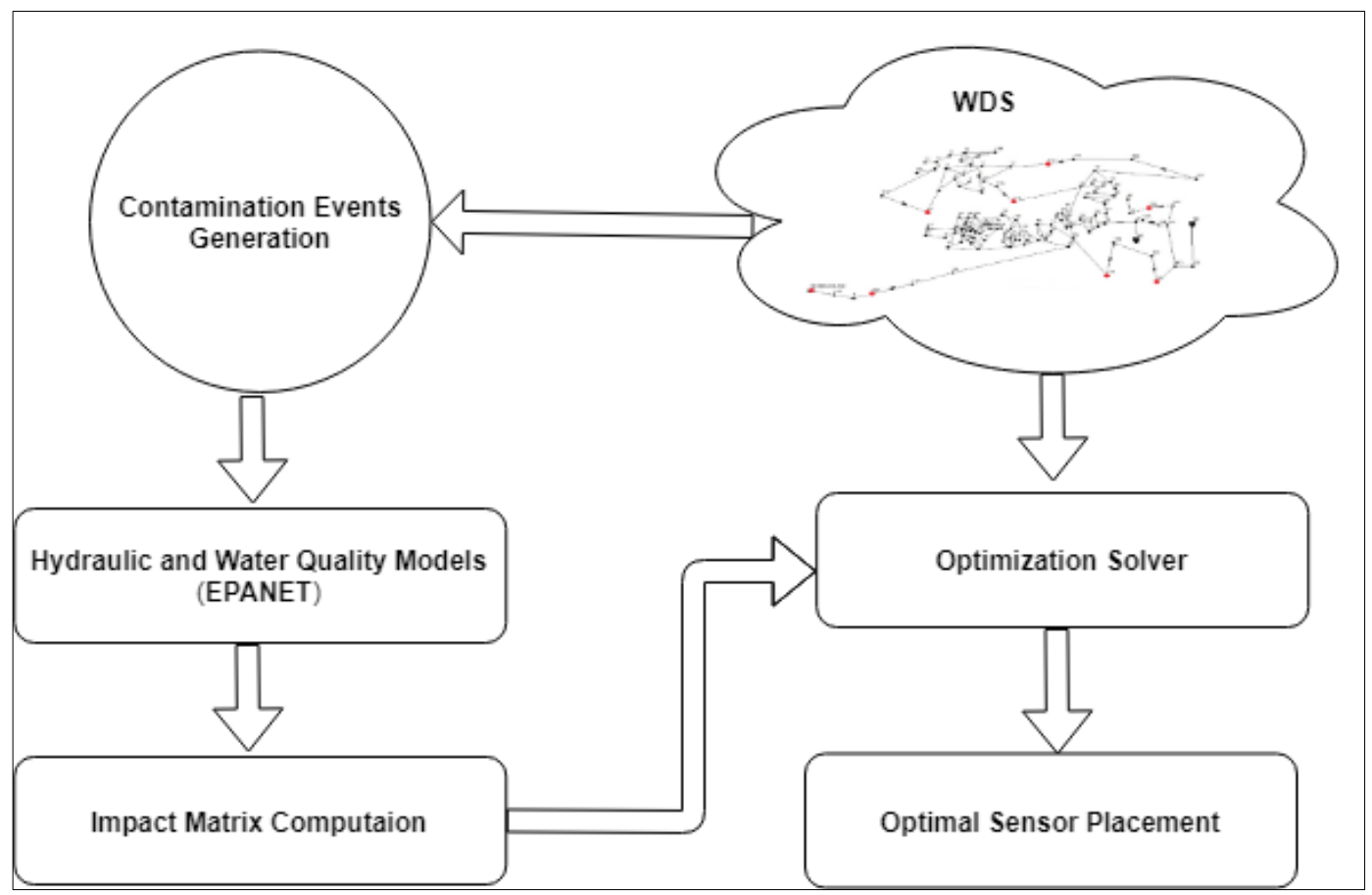

Figure 3 Methodology of the proposed approach

\subsection{Impact Matrix Computation}

In this work, two different objective functions (OF) have been used, which are: contaminant water consumed volume before contaminating detection, and timely detection of water contaminant. More detail about these objective functions is presented in the next two subs sections.

\subsubsection{The Volume of Contaminant Water (VCW)}

To calculate the amount of contaminated water was identified by any sensor, the following equation is used:

$$
V_{n e}(t)=\left\{\begin{array}{cc}
d_{i}(t) \times \Delta t & \text { if } C_{n e}(t) \geq C_{\text {min }} \\
0 & \text { otherwise }
\end{array}\right.
$$


Where $V_{n e}$ is the volume of the water at node $n$ for event $e, C_{\min }$ is the threshold hazard concentration, $C_{n e}$ is the concentration at node $\mathrm{n}$ for event $e . d_{i}(t)$ denotes the water demand at node $i$ at a time step, $\Delta t$ represents time step interval. Algorithm 1 shows the computation of the impact matrix, considering the volume of contaminant water for all event scenarios.

Algorithm 1: Calculating the volume of the contaminant water: Impact Matrix (VCW)



\subsubsection{Time detection (TD)}

The purpose of deploying water quality sensors in WDS is to effectively detect contamination events to reduce the risk of exposure to the public. In this work, our formulation models the placement of sensors $p$ in a set of nodes $\mathrm{P} \subseteq N, X P, X(j)$ means the $i$ th sensor in the layout of the sensor $X$. We use the expected time detection for all contaminants events $E$ as an optimization goal. For contaminant event $E(i)$, the detection time by sensor $X(j)$ is the elapsed time $t_{x(j)}$ from the start of the contaminant event to the first identified presence of a non-zero concentration of the contaminant. For a fixed number of sensors $p$, the time of the first detection is described as follows:

$$
t_{d}=\min _{j=1, \ldots, p}\left\langle t_{x(j)}\right\rangle
$$

The equation above is the objective of minimizing the expected value by calculating all contaminants events.

$$
\mathrm{t}=\min \frac{1}{\mathrm{E}} \sum_{\mathrm{i}=1}^{\mathrm{e}} \mathrm{t}_{\mathrm{d}}
$$

Where $t$ represents the mathematical expectation of the minimum detection time $t_{d}$. Since each contaminant event $e$ includes one injection that can occur at any node in the network, the number of all contaminant events is $\mathrm{E}$, if the sensor does not detect water contaminate event, $t_{d}$ is the simulation time.

\section{3 Optimization Solver (EA)}

The optimization formulas described above can be processed using several solvers for optimizing the placement of sensors that were used, including genetic algorithms, integer programming solvers, and local search. Other well-known methods, for example, some researchers simulated annealing and taboo searches. The choice of optimizer for determining the location of the sensor depends on several factors: a runtime available for optimization, available computer memory, the guarantee of performance for the final solution. Integer programming (IP) solvers can ensure that the best possible location for the sensor is found, that is, a place that minimizes the risk of water contamination. However, it is known that IP solvers have difficulty solving large applications; in large tasks, they can work for long hours and require a lot of memory. In contrast, heuristic optimizers, such as genetic algorithms or local search methods, are typically unable to guarantee that the final solution is almost optimal. But in practice, it is known that these methods quickly find practically optimal solutions. In this work, an evolutionary algorithm (EA) is used to represent the optimization solver. The essential descriptions of EA and how it applied in our case studies are described in algorithms (2 and 3). 
Algorithm 2: The main description of $\boldsymbol{E A}$

1: Generate [Pop [0]]; Generate the initial Population.

2: $\mathrm{T} \leftarrow 0$

3: while (the target performance NOT Obtained) do

4: Evaluate Pop (t); Evaluate the fitness of each individual

5: Pop' $(\mathrm{T}) \leftarrow$ Select pop $(\mathrm{t})$; select the best-fit individual

6: pop" $(\mathrm{t}) \leftarrow$ Apply variation operations; Crossover and Mutation on Pop' $(\mathrm{t})$

7: pop $(\mathrm{t}+1) \leftarrow$ Replace [ pop' $(\mathrm{t})$, pop" $(\mathrm{t})]$.

8: $\mathrm{T} \leftarrow \mathrm{t}+1$

9: end while

In algorithm 2, the primary function of the evaluation algorithm is presented. The motivation for using this algorithm is that the EA is designed to mimic natural evolution. This algorithm has three principles that formed the foundation of development: population of individuals, mutation, and fitness. A population can be reproduced or died, and this determines by fitness. The population can mutate by amending a bit in its properties to produce a new generation. EA has been applied in the two different case-studies (BWSN and Madrid) to select the optimal locations of the sensors. EA operates by generating the initial population randomly, where the population represents the potential solutions, and then the fitness of each individual is evaluated. The fitness is represented by the impact matrix (see section 3.2) that uses two objective functions to assess the performance of the algorithm: VCW and TD. In BWSN case-study, we applied only VCW with the fitness function where the location with high volume will be selected for the new population while the places with low volume will be ignored (died). The details of how VCW is calculated is explained in section 3.2.1. In Madrid case-study, VCW and TD are applied where the fitness with TD selects the locations that have minimum values of time detections and ignore the others. For the mutation, we have added Gaussian noise to each offspring in which mutation must not push the children beyond locations number (our target solutions). The implementation of EA with two case studies is presented in Algorithm 3.

\section{Algorithm 3: The implementation of $\boldsymbol{E A}$ in our case-studies}

1: Carry out the Hydraulic analysis of network

2: Consider each node as a candidate location for first sensor placement

3: Determine the Objective Function (OF) values for all available alternative sensor locations

4: Select the node combination of a node for sensor placement having target value of OF

5: if (Target value of OF obtained) and (Number of sensor location gained) then

6: $\quad$ Go to END!

7: else

8: Determine the available sensor by adding one more sensor location to already

Selected location.

9: $\quad$ Go to step 4

\section{Case Studies}

The sensor placement method proposed in this paper has been tested and investigated using two different case studies. Firstly, the Battle of the Water Sensor Network (BWSN) case-study network, where we examined how the water contaminant spread in the system. We investigated how the deployed sensors adapt their locations against numbers and locations of pollutant water sources (i.e., single or two contaminant sources at the runtime). Secondly, based on a real case study from Madrid 
(Spain). We have tested the ability of the deployed sensors using our approach to detect all water contaminants.

\subsection{Battle of the Water Sensor Network (BWSN)}

The BWSN shown in Figure 4 includes one reservoir, two pumps, eight valves, one hundred twentysix junctions, and one hundred sixty-eight pipes. Each contamination event considered in this study has a concentration of $10 \mathrm{mg} / \mathrm{L}$ and injection time of two hours (between the start and stop time). The source of the contamination event could be single or multiple (i.e., 2 locations). This case was used to test the ability of the proposed algorithm to adapt the positions of deployed sensors based on the number of contaminant sources and will present the contamination generation in the network as colored maps that include deployed sensors.

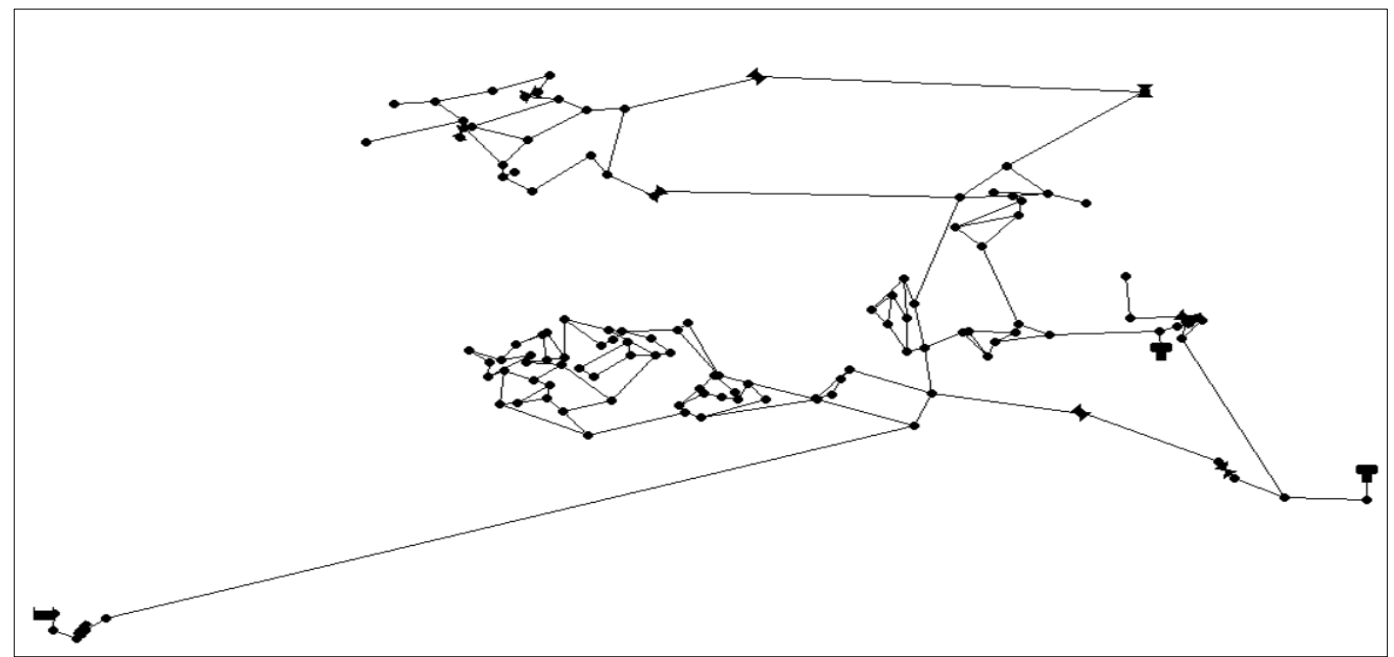

Figure 4 BWSN case-study

\subsection{Madrid Capital}

This real case study denotes a part of the town (city centre) of Madrid Capital, Spain, as shown in Figure 5. It includes one reservoir, two tanks, two pumps, fifty-five valves, four hundred and forty pipes, and one thousand junctions. This case study was being used to test the capacity of the deployed sensors to detect all water contaminant events that may occur at any location based on two different objectives, which are the volume of contaminated water and time detection.

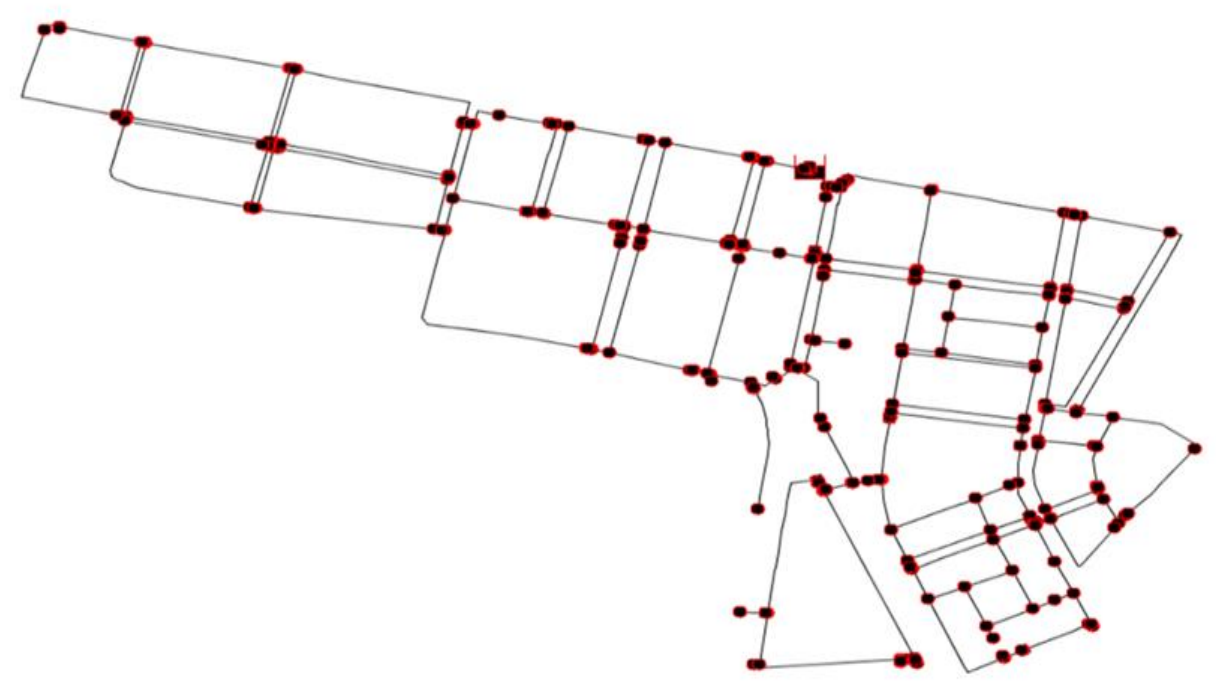

Figure 5 Madrid city case-study 


\section{Results and Analysis}

\subsection{Results from BWSN Case Study}

The results obtained from the BWSN case study is presented in this section, the following scenario is considered. Firstly, contamination started to spread in the network from TANK-131 (see Figure 6) at time zero of the 50 hours EPANET simulation time. At this point, a single source of contamination is suspected, and the optimally placed sensors located injunctions 10, 17, 23, and 32 (see Figure 7) enables detecting that contamination event. The effectiveness of that sensor placement can be inferred by looking at the contamination regions in the WDS after 16 hours of simulations shown in Figure 6. This map shows five different colours in which blue represents a region with no contamination, while the red represents regions with high diseases. After that, a second source of the pollution is suspected (simulated as a contamination event that started to spread in the network from (RESERVOIR-129) as shown in Figure 8. Therefore, the sensors should move to the optimal locations injunctions 10, 34, 53, and 118 (see Figure 9) to more effectively detect the two-source contamination event. Similar to what is shown in Figure 6, Figure 8 demonstrates the effectiveness of that sensor placement by showing the contamination regions after 30 hours of simulations.

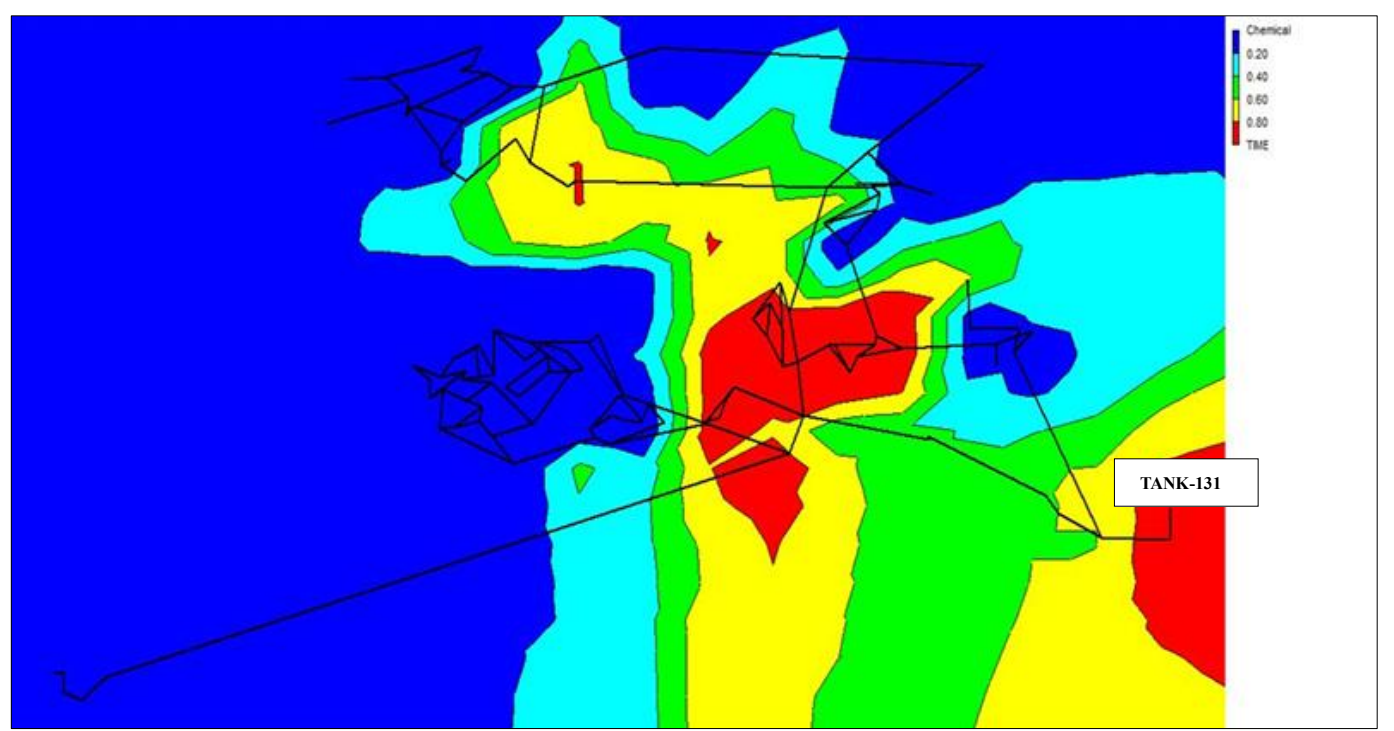

Figure 6 Contamination spread in the network from single contaminated source

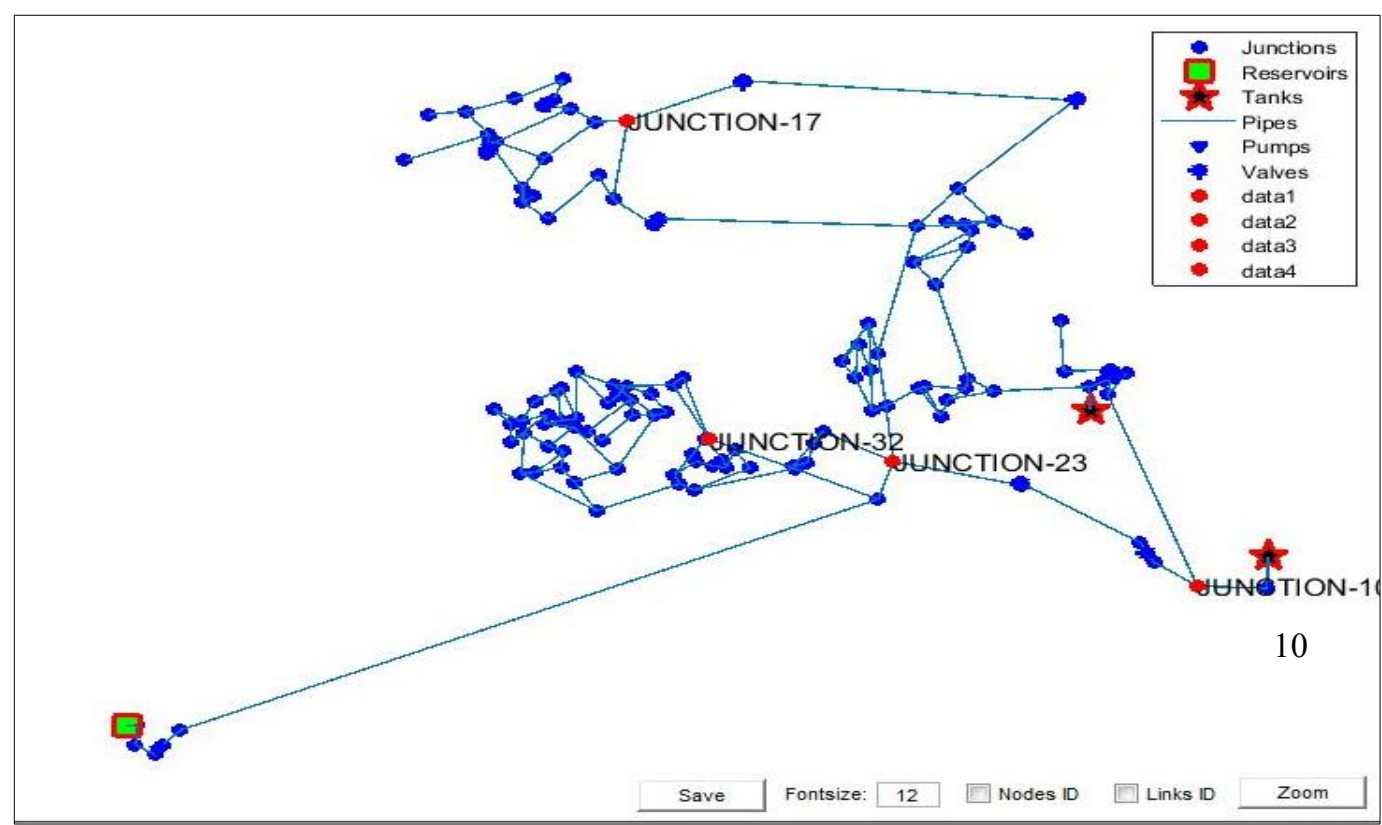

Figure 7 Optimal locations of sensors with single contaminations source 


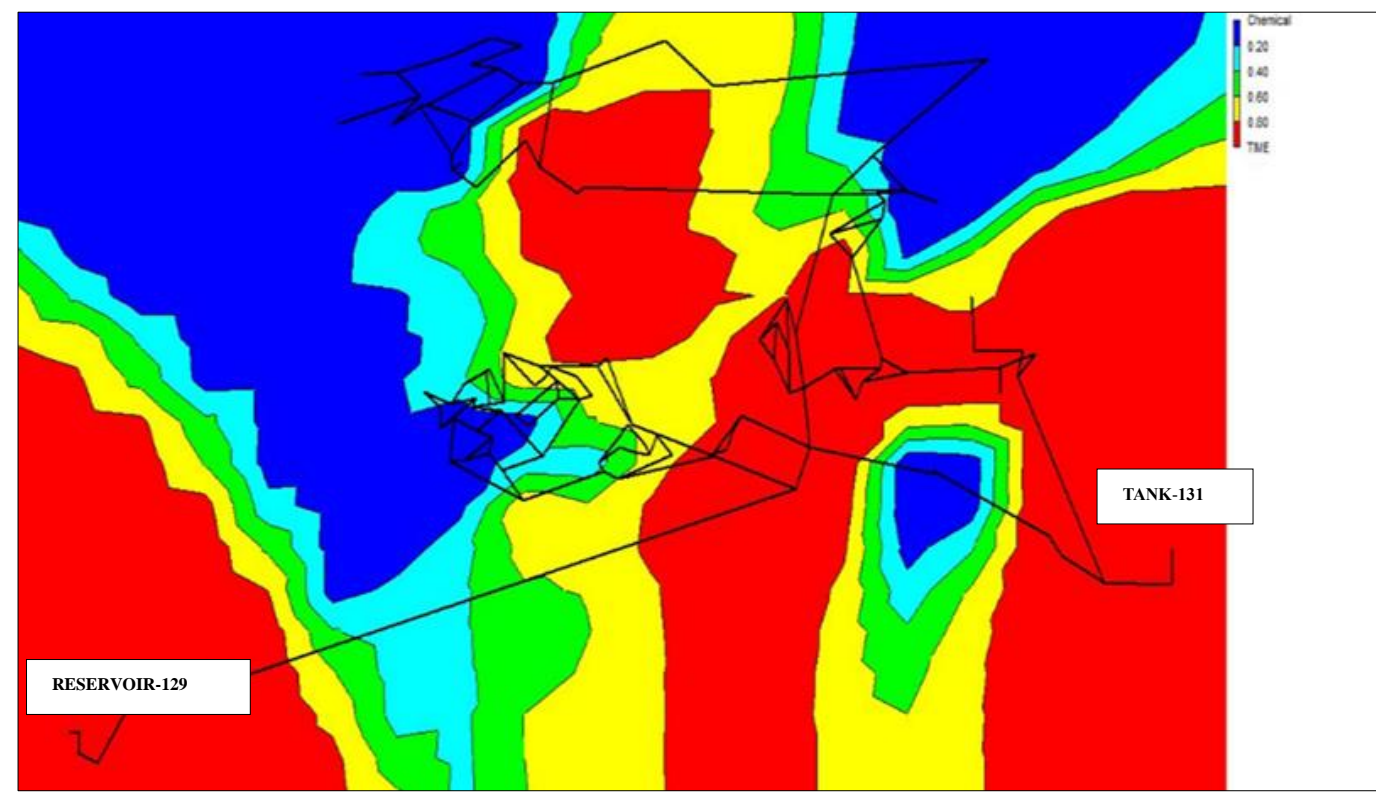

Figure 8 Contamination spread in the network from two contaminated sources

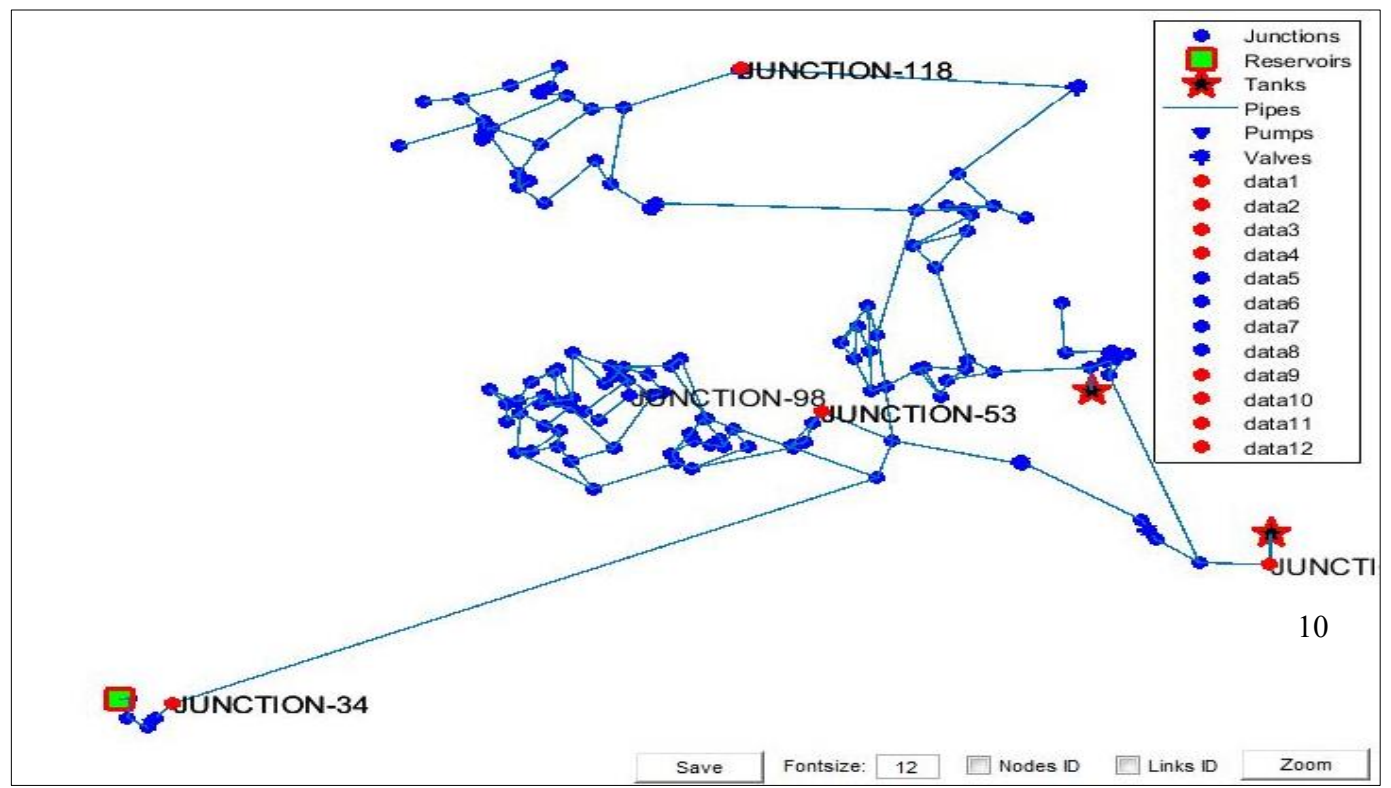

Figure 9 Optimal locations of sensors with two contaminations sources

\subsection{Results from Madrid Case Study}

The results from the Madrid case study described above are presented in this section. Start to show how the water contamination spread in the network for 24 hours. Figure 10 shows the regions that include water contamination labelled by red and the areas without any contamination labelled by blue. We have tested our approach in the Madrid case study utilizing different experimental settings for timely detection and volume of contaminated consumed water as objective metrics for optimizer solver. In the first experiment, we have used ten sensors that have to be deployed in the study area; the volume of water consumed has been used as an objective function for optimizer solver. Figure 11 shows the optimal locations of ten sensors that cover the network. To justify the capacity of these sensors to detect contaminates, Figure 12 represents the ten sensors with ten different colors; each color represents the coverage of each sensor to detect contaminates. We can notice from Figure 12 that sensor locations cover most of the study area, which means that it can detect all water contaminants. Besides, some regions labeled with black are represented undetected areas of water contamination. 


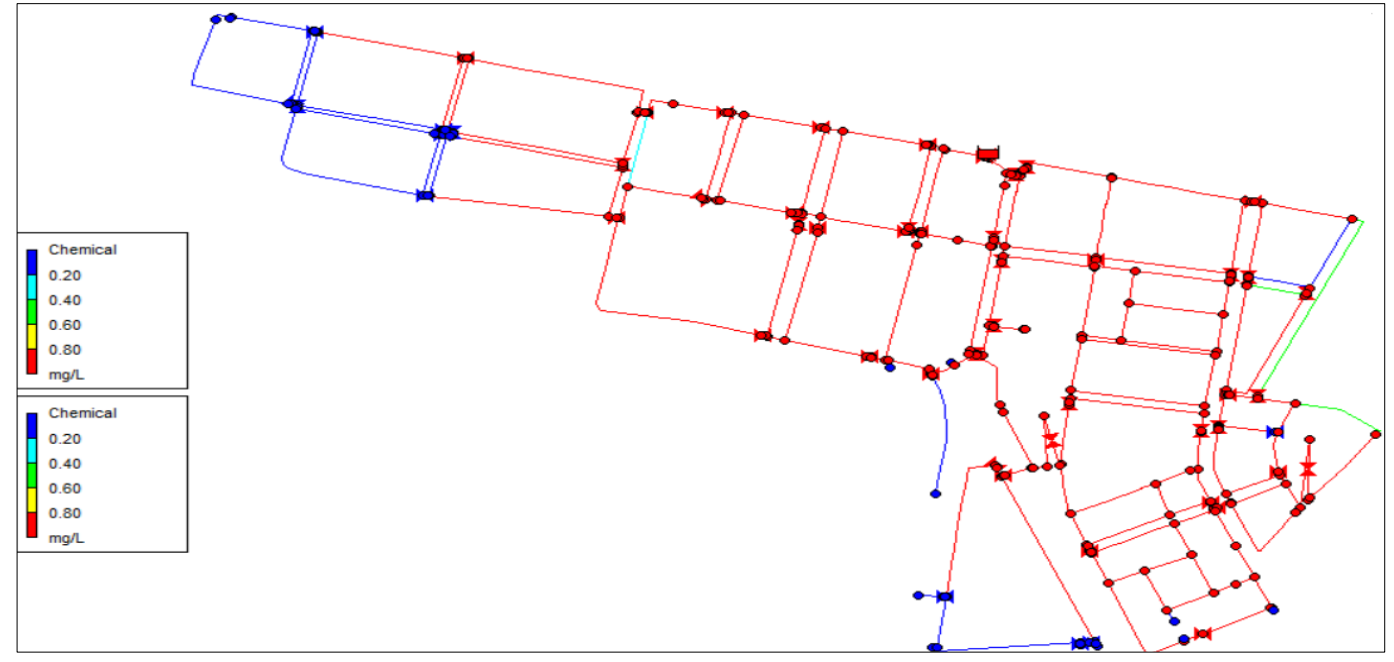

Figure 10 Water contamination spread in the network after 24 hours

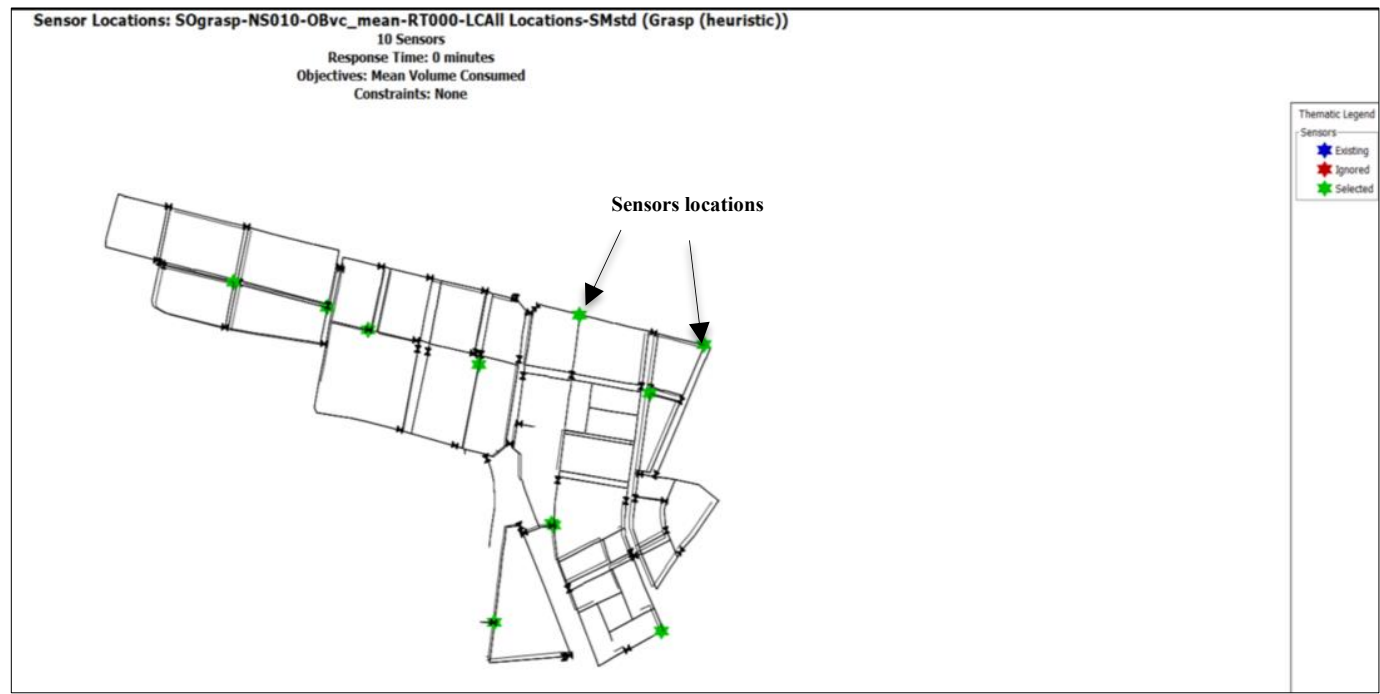

Figure 11 Sensor locations: ten sensors with volume contaminant water

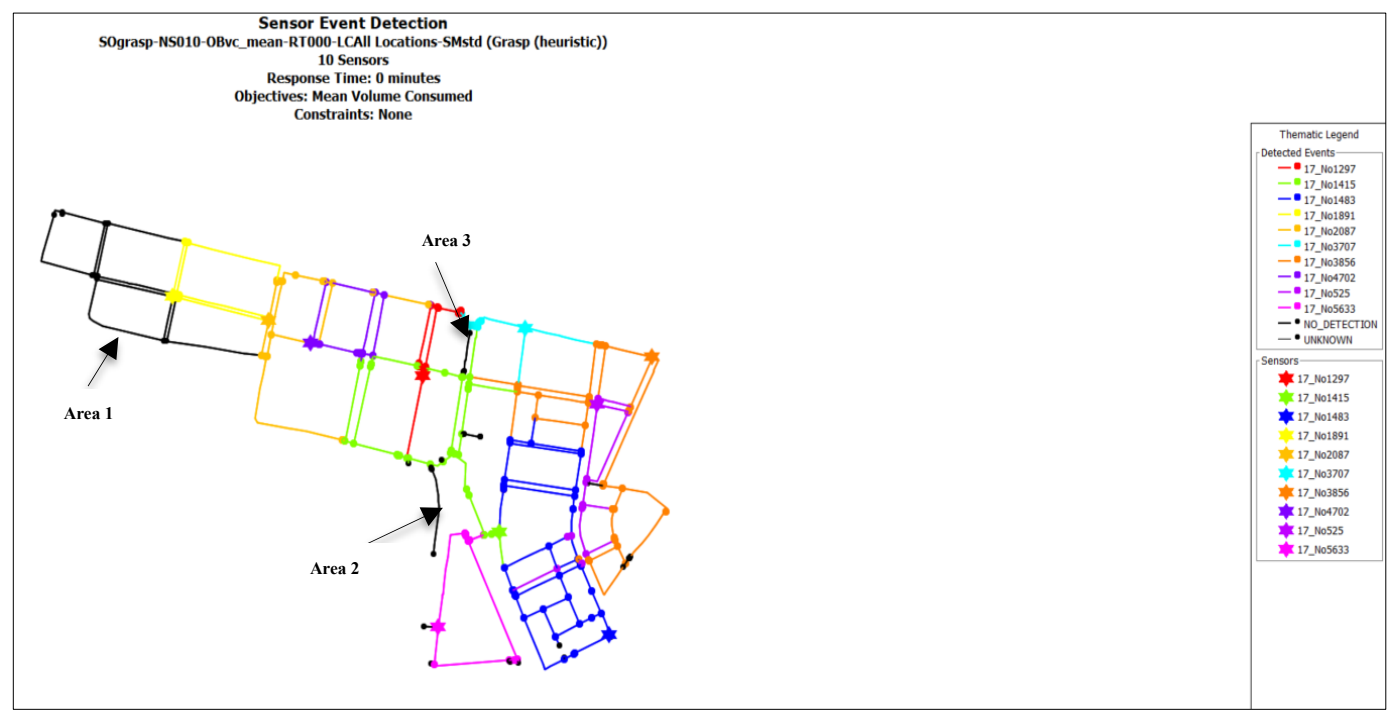

Figure 12 Sensor event detection: (links and nodes: ten sensors with VCW) 
To investigate how the deployed sensors cover the areas based on the volume of the consumed water, firstly, looking to Figure 12 again, the area in the most left-hand side does not cover directly by any sensor (labelled by Area 1) this is because this area has a low number of houses which leads to low water demand. As the volume of contaminated water is used as an objective function in this experiment, these areas with low water demand will get low priority to cover directly by the deployed sensors. Secondly, another undetected area can be noticed in Figure 12, either the isolated links (links not connected to the network form both sides labelled by Area 2) or the links that have connected two points in parallel with other links (marked by Area 3). This is because either the area is separated from the whole network, which means that the water flow and demand are low or the links are connected in parallel with others, which means that all water flow and demand pass through the other loop.

The results that represented the optimal locations of the ten sensors deployed using different constraints are shown in Figure 13. In this experiment, we have used ten sensors and time detection as objective functions. To justify the positions of these sensors to the event detection, Figure 14 shows the ability of these sensors with ten different colours; each colour represents the coverage of each sensor for detecting the contamination. We can notice that the sensor locations are changed compared to the previous ones shown in Figure 11. This is because detection time is used in this experiment as objective functions for the optimizer, so the sensor location does not depend on water demand. However, the optimal locations of this sensor aim to detect the contaminant as fast as possible. From Figure 14, we can also notice that the whole study area (water network) is covered directly by the deployed sensors except the isolated regions (ex. labelled by Area 1) that ignored from the leading system and considered as a separate network.

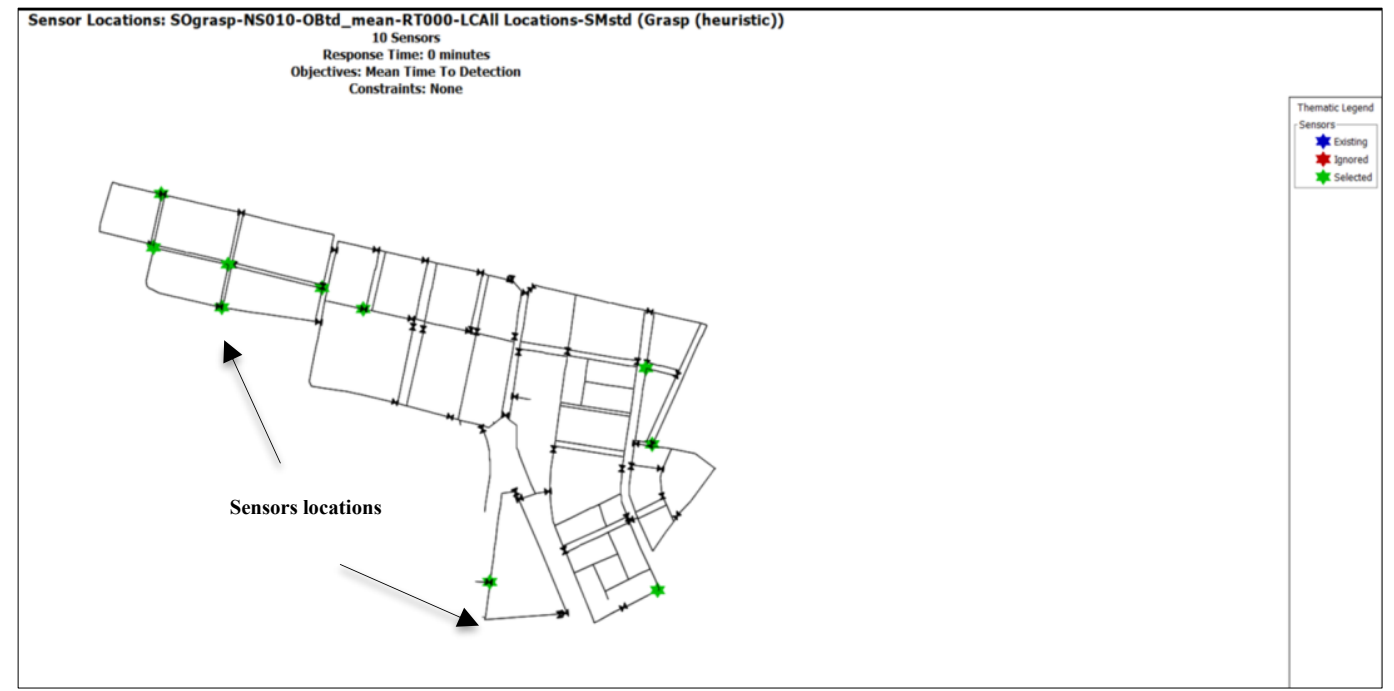

Figure 13 Sensor locations: tens sensors with time detections

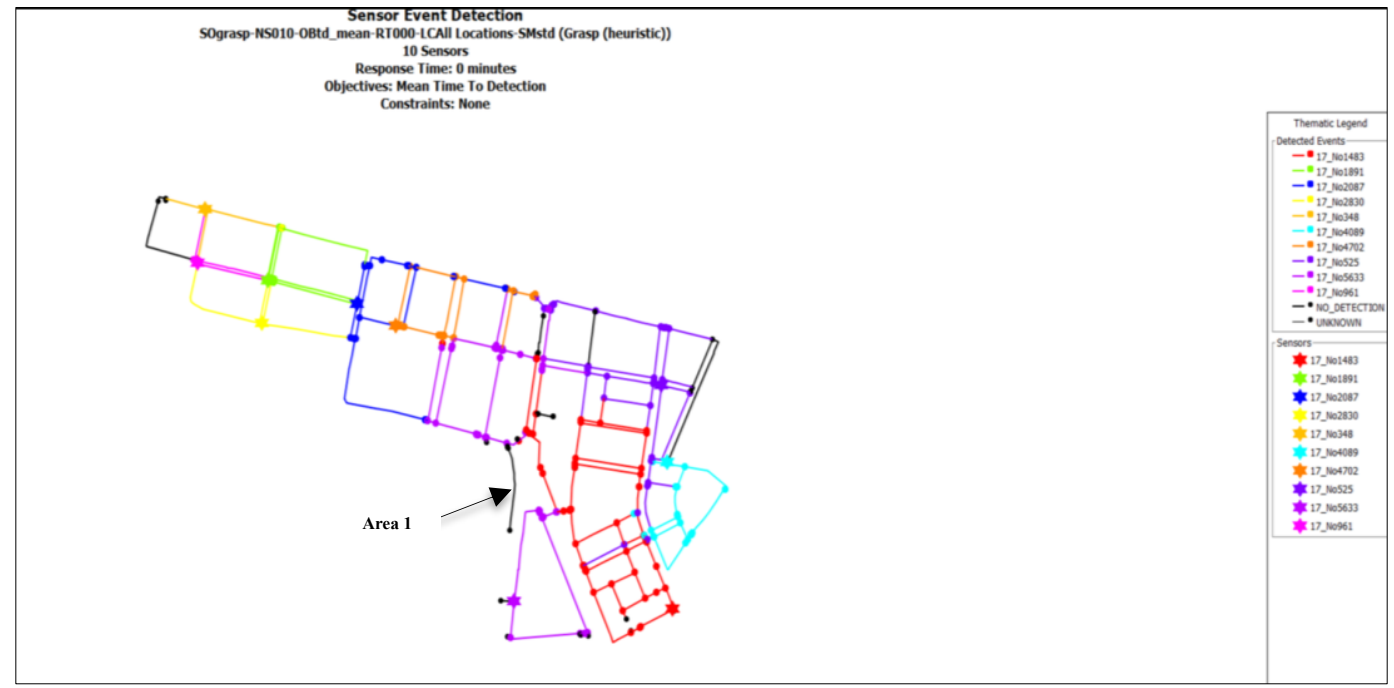

Figure 14 Sensor event detection: (links and nodes: ten sensors with TD) 


\section{Conclusion}

In this paper, a dynamic approach of water quality sensor placement that uses to detect the water contaminations in near real-time is proposed. The proposed method generates a multiple set of water contamination scenarios in several locations selected randomly in the WDS. The multiple optimization objective functions (volume of contamination water and time of water contamination detection) have been formulated and used with EA as optimization functions. Then, the sensor placement optimization solver is operated to find the optimal sensor placements. The proposed method has been evaluated using different case studies BWSN and Madrid. In the BWSN casestudy, we have tested the ability of our approach to adapt the sensor locations based on the number and locations of suspected contamination sources. In Madrid Capita case-study, we have examined the strength of the proposed approach to maximize the coverage of detecting water contaminations using a low number of water quality sensors. The results show that the proposed method has achieved the goals of identifying the water contaminations while fulfilling the requirements of the near real-time monitoring using a low-cost sensor deployment.

For future work, we aim to study the impact of human health from water contamination and how the proposed approach presented in this work is helping to reduce water contamination impact. The health impact can be analyzed, by computing the number of people got effect from the water contamination after spreading in water distribution networks for a few hours in term of fatality, infected, and disease.

\section{Acknowledgment}

This research is supported by the European Union's Horizon 2020 research and innovation program Under the Marie Skłodowska-Curie-Innovative Training Networks (ITN)-IoT4Win (765921).

\section{References}

Adu-Manu KS, Tapparello C, Heinzelman W, Katsriku FA, Abdulai J-D (2017) Water quality monitoring using wireless sensor networks: Current trends and future research directions ACM Transactions on Sensor Networks (TOSN) 13:4

Angelakis A, Snyder S (2015) Wastewater treatment and reuse: Past, present, and future. Multidisciplinary Digital Publishing Institute,

Banna MH, Imran S, Francisque A, Najjaran H, Sadiq R, Rodriguez M, Hoorfar M (2014) On-line drinking water quality monitoring: review on available and emerging technologies Critical Reviews in Environmental Science and Technology 44:1370-1421

Barabde M, Danve S (2015) Real time water quality monitoring system International Journal of Innovative Research in Computer and Communication Engineering 3:5064-5069

Capodaglio AG (2017) In-stream detection of waterborne priority pollutants, and applications in drinking water contaminant warning systems Water Science and Technology: Water Supply 17:707-725

Chang, K., Gao, J.L., Wu, W.Y. and Yuan, Y.X., (2011) Water quality comprehensive evaluation method for large water distribution network based on clustering analysis. Journal of Hydroinformatics, 13(3): 390-400.

Chen J, Li T, Shu T, De Silva CW (2018) Rapidly-exploring tree with linear reduction: A nearoptimal approach for spatiotemporal sensor deployment in aquatic fields using minimal sensor nodes IEEE Sensors Journal 18:10225-10239

Chen Y, Han D (2018) Water quality monitoring in smart city: A pilot project Automation in Construction 89:307-316

Cloete NA, Malekian R, Nair L (2016) Design of smart sensors for real-time water quality monitoring IEEE Access 4:3975-3990

Cotruvo J, Craun GF, Hearne N (2018) Providing safe drinking water in small systems: Technology, operations, and economics. Routledge, 
De Vera GA, Wert EC (2019) Using discrete and on-line ATP measurements to evaluate regrowth potential following ozonation and (non) biological drinking water treatment Water research 154:377-386

De Winter C, Palleti VR, Worm D, Kooij R (2019) Optimal placement of imperfect water quality sensors in water distribution networks Computers \& Chemical Engineering 121:200-211

Dong J, Wang G, Yan H, Xu J, Zhang X (2015) A survey of smart water quality monitoring system Environmental Science and Pollution Research 22:4893-4906

Du Plessis A (2017) Global Water Quality Challenges. In: Freshwater Challenges of South Africa and its Upper Vaal River. Springer, pp 13-44

Gheisi A, Forsyth M, Naser G (2016) Water distribution systems reliability: A review of research literature Journal of Water Resources Planning and Management 142:04016047

Hall J, Zaffiro AD, Marx RB, Kefauver PC, Krishnan ER, Haught RC, Herrmann JG (2007) OnLine water quality parameters as indicators of distribution system contamination Journal American Water Works Association 99:66-77

Huang X, Yi J, Chen S, Zhu X (2015) A wireless sensor network-based approach with decision support for monitoring lake water quality Sensors 15:29273-29296

Kara S, Karadirek IE, Muhammetoglu A, Muhammetoglu H (2016) Real time monitoring and control in water distribution systems for improving operational efficiency Desalination and water treatment 57:11506-11519

Karadirek I, Kara S, Muhammetoglu A, Muhammetoglu H, Soyupak S (2016) Management of chlorine dosing rates in urban water distribution networks using on-line continuous monitoring and modeling Urban Water Journal 13:345-359

Karem K, Issa A, SOLIMAN A-H, ALY H (2017) Inline Water Quality Monitoring System (IWQMS)

Liu G, Zhang Y, Knibbe W-J, Feng C, Liu W, Medema G, van der Meer W (2017) Potential impacts of changing supply-water quality on drinking water distribution: A review Water research 116:135-148

Menon KU, Divya P, Ramesh MV Wireless sensor network for river water quality monitoring in India. In: 2012 Third International Conference on Computing, Communication and Networking Technologies (ICCCNT'12), 2012. IEEE, pp 1-7

Parra L, Sendra S, García L, Lloret J (2018) Design and deployment of low-cost sensors for monitoring the water quality and fish behavior in aquaculture tanks during the feeding process Sensors 18:750

Pule M, Yahya A, Chuma J (2016) A wireless sensor network solution for monitoring water quality in Botswana. In: 2016 3rd National Foundation for Science and Technology Development Conference on Information and Computer Science (NICS), 2016. IEEE, pp 12-16

Rathi S, Gupta R (2014) Sensor placement methods for contamination detection in water distribution networks: A review Procedia Engineering 89:181-188

Richardson SD (2011) Environmental mass spectrometry: emerging contaminants and current issues Analytical Chemistry 84:747-778

Rock CM, Brassill N, Dery JL, Carr D, McLain JE, Bright KR, Gerba CP (2019) Review of water quality criteria for water reuse and risk-based implications for irrigated produce under the FDA Food Safety Modernization Act, produce safety rule Environmental research 172:616629

Ross C, Petzold H, Penner A, Ali G (2015) Comparison of sampling strategies for monitoring water quality in mesoscale Canadian Prairie watersheds Environmental monitoring and assessment 187:395

Sela L, Amin S (2018) Robust sensor placement for pipeline monitoring: Mixed integer and greedy optimization Advanced Engineering Informatics 36:55-63

Shahra E, Wu W (2019) Dynamic Water Quality Sensor Placement Using Metaheuristic Algorithm on Water Distribution System

Shahra EQ, Wu W, Romano M (2019) Considerations on the deployment of heterogeneous IoT devices for smart water networks. In: IEEE SmartWorld, Ubiquitous Intelligence \& Computing, Advanced \& Trusted Computing, Scalable Computing \& Communications, Cloud \& Big Data Computing, Internet of People and Smart City Innovation (SmartWorld/SCALCOM/UIC/ATC/CBDCom/IOP/SCI), 2019. IEEE, pp 791-796

Sheltami TR, Shahra EQ, Shakshuki EM (2017) Perfomance comparison of three localization protocols in WSN using Cooja Journal of Ambient Intelligence and Humanized Computing 8:373-382

Storey MV, Van der Gaag B, Burns BP (2011) Advances in on-line drinking water quality monitoring and early warning systems Water research 45:741-747 
Thompson K, Kadiyala R (2014) Protecting water quality and public health using a smart grid Procedia Engineering 70:1649-1658

Tuna G, Arkoc O, Gulez K (2013) Continuous monitoring of water quality using portable and lowcost approaches International Journal of Distributed Sensor Networks 9:249598

Vinod G, Peter A, Rao I, Babu Y (2018) IoT based Water Quality Monitoring System Using WSN Indian Journal of Public Health Research \& Development 9

Yang Y, Gu X, Te SH, Goh SG, Mani K, He Y, Gin KY-H (2019) Occurrence and distribution of viruses and picoplankton in tropical freshwater bodies determined by flow cytometry Water research 149:342-350

Zaev E, Babunski D, Tuneski (2016) A SCADA system for real-time measuring and evaluation of river water quality. In: 5th Mediterranean Conference on Embedded Computing (MECO), 2016. IEEE, pp 83-86

Zeng D, Gu L, Lian L, Guo S, Yao H, Hu J (2016) On cost-efficient sensor placement for contaminant detection in water distribution systems IEEE Transactions on Industrial Informatics 12:2177-2185

Zhuiykov S (2012) Solid-state sensors monitoring parameters of water quality for the next generation of wireless sensor networks Sensors and Actuators B: Chemical 161:1-20

Zulkifli SN, Rahim HA, Lau W-J (2018) Detection of contaminants in water supply: A review on state-of-the-art monitoring technologies and their applications Sensors and Actuators B: Chemical 255:2657-2689 